\title{
Persistent damage to Enterocytozoon bieneusi, with persistent symptomatic relief, after combined furazolidone and albendazole in AIDS patients
}

D Dionisio, L Ibba Manneschi, S Di Lollo, A Orsi, G Sterrantino, F Leoncini, M Pozzi, M A Vinattieri, A Tani, A Papucci

Infectious Diseases Unit, Pistoia Hospital, Pistoia, Italy D Dionisio

Department of Human Anatomy and Histology, University of Florence, Italy L Ibba Manneschi A Tani

Institute of Pathology, University of Florence, Italy

S Di Lollo

A Papucci

Laboratory for Microbiology and Virology, Careggi Hospital, Florence, Italy

A Orsi

Infectious Diseases Unit, Careggi Hospital, Florence, Italy G Sterrantino F Leoncini

M Pozzi

Infectious Diseases Unit, Prato Hospital, Prato, Italy

M A Vinattieri

Correspondence to: Dr Daniele Dionisio, Infectious Diseases Unit, Pistoia Hospital, Piazza Giovanni XXIII, 51100 Pistoia, Italy.

Accepted for publication 13 May 1998

\begin{abstract}
Aim-To investigate morphological changes in Enterocytozoon bieneusi and the duration of symptomatic relief after combination treatment with furazolidone and albendazole in AIDS patients.

Methods-Four severely immunocompromised AIDS patients with symptomatic $E$ bieneusi infection of the gut received an 18 day course of combined furazolidone and albendazole $(500+800 \mathrm{mg}$ daily). All patients were monitored for parasite shedding in stool by light microscopy at the end of treatment and monthly during follow up. At the end of treatment, duodenal biopsy specimens obtained from three patients were studied by transmission electron microscopy by two pathologists blind to the patients' treatment or clinical outcome. Duodenal biopsy specimens obtained from one of the patients two months after completion of treatment were also studied electronmicroscopically. Results-All patients had long lasting symptomatic relief, with a major decrease-or transient absence-of spore shedding in stools from completion of treatment. After treatment, changes in faecal spores were persistently found by light microscopy in all cases, and there was evidence of both a substantial decrease in parasite load and ultrastructural damage in the parasite in all biopsy specimens. The treatment was well tolerated, and no patient had clinical or parasitological relapse during follow up (up to 15 months).

Conclusions-The long lasting symptomatic relief observed in all four treated patients correlated with the persistent decrease in parasite load both in tissue and in stool, and with the morphological changes observed in the life cycle of the protozoan. These data suggest that combined treatment with furazolidone and albendazole is active against $E$ bieneusi and may result in lasting remission even in severely immunocompromised patients. (F Clin Pathol 1998;51:731-736)
\end{abstract}

Keywords: Enterocytozoon bieneusi; furazolidone; albendazole; immune deficiency

Enterocytozoon bieneusi is the most commonly found microsporidium in chronic diarrhea and wasting in AIDS patients. ${ }^{12}$ No established treatment is currently available because none of the drugs tested can eradicate the infection (with the exception, perhaps, of fumagillin, which has toxic effects that make it unsuitable for clinical use $\mathrm{e}^{2 \mathrm{~b}}$ ) or significantly inhibit the life cycle of the parasite; moreover, relapses rapidly occur after discontinuation of treatment. ${ }^{13-5}$ Also albendazole, which is considered the drug of choice for microsporidiosis caused by Encephalitozoon spp, ${ }^{6-9}$ was reported to be poorly and variably effective against $E$ bieneusi, although changes in the parasite were described after treatment. ${ }^{10-12}$

Recently we reported both symptomatic relief and light and electron microscopic changes in $E$ bieneusi, with decreased parasite shedding in the stool, after treatment with furazolidone in six patients with AIDS. ${ }^{13}$ The infection, however, was not eradicated by the schedules of treatment used, and two patients had clinical and parasitological relapse two months after treatment was discontinued.

A search for more lasting results led us to use a combination of furazolidone and albendazole to treat four AIDS patients with symptomatic E bieneusi infection of the gut. The rationale of this approach lies in the fact that the mechanism of action of these drugs seems complementary, being directed at different metabolic pathways in the parasite. Furazolidone probably interferes with the synthesis of nucleic acids by an action on the Krebs cycle, while albendazole, which inhibits tubulin polymerisation into microtubules of parasites, ${ }^{14-16}$ may affect the precursors of polar tubes of E bieneusi.

In this study our aim was to confirm the results obtained using furazolidone alone, ${ }^{13}$ and to evaluate the ability of combined furazolidone and albendazole to produce persistent clinical and parasitological remission without the need for maintenance treatment.

\section{Methods}

We enrolled four HIV positive drug misusing patients (three male, one female) with symptomatic $E$ bieneusi infection of the gut. The patients were aged between 31 and 35 years. All had AIDS at the time of study entry; according to the 1993 CDC revised classification system, two cases belonged to the $\mathrm{C}_{3}$ stage, while the others to the $B_{3}$ stage. The mean CD4+T cell count was $7.7 \times 10^{6} /$ litre (range 4 to 11). All patients had chronic diarrhoea (for at least two months), with a range of two to five watery stools a day (mean four). Weight loss ranged between 3 and $8 \mathrm{~kg}$ (mean 5.5). 
Stool were processed according to the methods of Weber et al and of Van Gool et al to detect microsporidia. ${ }^{17-19}$ The amount of spore shedding was evaluated by a semiquantitative criterion on 100 microscopic fields: spores were defined as very frequent if present in every field, frequent if present in more than 50 but fewer than 100 fields, rare if present in fewer than 50 fields, and very rare if sporadically present.

Before study entry, frequent to very frequent spores of microsporidia were present in stools of all patients. Stool samples were also examined for bacteria, Clostridium difficile toxin, mycobacteria, eggs and parasites, adenoviruses, and rotaviruses, with negative results.

Transmission electron microscopy (TEM) was performed on endoscopic biopsy specimens taken from the third part of duodenum before treatment. At least two samples from each patient were processed. Specimens were fixed with glutaraldehyde $2.5 \%$ in cacodylate buffer $(\mathrm{pH}$ 7.4) for three to four hours at room temperature, washed in the same buffer, and postfixed in osmium tetroxide in $1 \%$ Milloning buffer (SIC, Rome, Italy) for one hour at room temperature. After dehydration through graded acetone series, the specimens passed through propylene oxide and were then embedded in Epon 812 . Semithin sections $(3 \mu \mathrm{m})$ were cut, stained with toluidine blue-sodium tetraborate, and studied by light microscopy. About 100 ultrathin sections were obtained from all the selected specimens and were stained with uranyl acetate and alkaline bismuth subnitrate. For each section at least three random fields were examined by TEM. Care was taken to evaluate only areas away from the borders of the biopsy specimen to avoid traumatic artefact.

All patients underwent a baseline evaluation that included full blood cell count, urine analysis, electrolytes, liver function tests, and abdominal ultrasonography.

Combination treatment with furazolidone (100 mg tablet five times daily) and albendazole (400 mg tablet twice daily) was given orally to all the patients for 18 days. Written informed consent was obtained before treatment. No concomitant antidiarrhoeal drugs were allowed. Clinical assessment and haema- tological and liver function tests were monitored after 10 days and at the end of treatment. Adverse events were evaluated.

Three days after completion of treatment, upper endoscopy was performed in three patients who gave their consent. One of these patients also underwent endoscopy two months after discontinuation of treatment. In all cases, at least two biopsy specimens were taken from the third part of duodenum, and were processed as described above. The ultrathin sections obtained were examined by TEM by two pathologists blind to the patients' treatment or clinical outcome.

In all patients spore shedding in the stool was monitored by Weber's method at the end of treatment and monthly during follow up (one stool sample a day for three days). Daily stool frequency and body weight were charted, and the CD4+T cell count was repeated every four months after discontinuation of treatment. The mean duration of follow up was eight months (range four to 15).

A complete response was defined as cessation of diarrhoea (stool frequency less than twice a day), stabilisation of body weight, and a major decrease (or clearance) of spore shedding in the stools for more than three months. A partial response was defined as improvement in baseline stool frequency, with continuing weight loss, and slight or no decrease in spore shedding in stool.

\section{Results}

CLINICAL RESPONSE

All patients showed a complete response, with remission of symptoms and a major decrease or absence of spore shedding in the stools from the time of completion of treatment. The drugs were well tolerated, although, probably as a side effect of furazolidone, ${ }^{20}$ there was a slight worsening of diarrhoea in all patients during treatment. This was promptly reversed when treatment was discontinued, and there were no alterations in the laboratory test results attributable to the drugs used.

Over the follow up period no patient had a relapse, either clinical or parasitological, despite the fact that no maintenance treatment was given and no improvement in CD4+T cell count was documented.

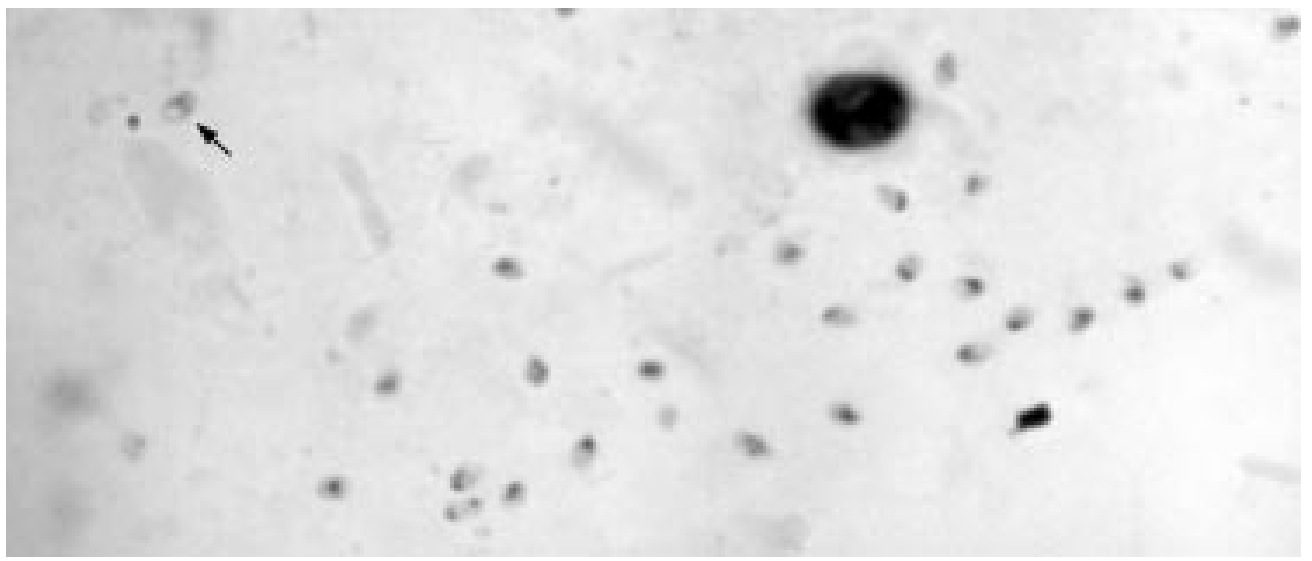

Figure 1 Microsporidian spores (stool specimen after treatment). Many spores are evident, almost all showing a pronounced red stained clot. A normal spore is seen (arrow). (Magnification $\times 795$.) 

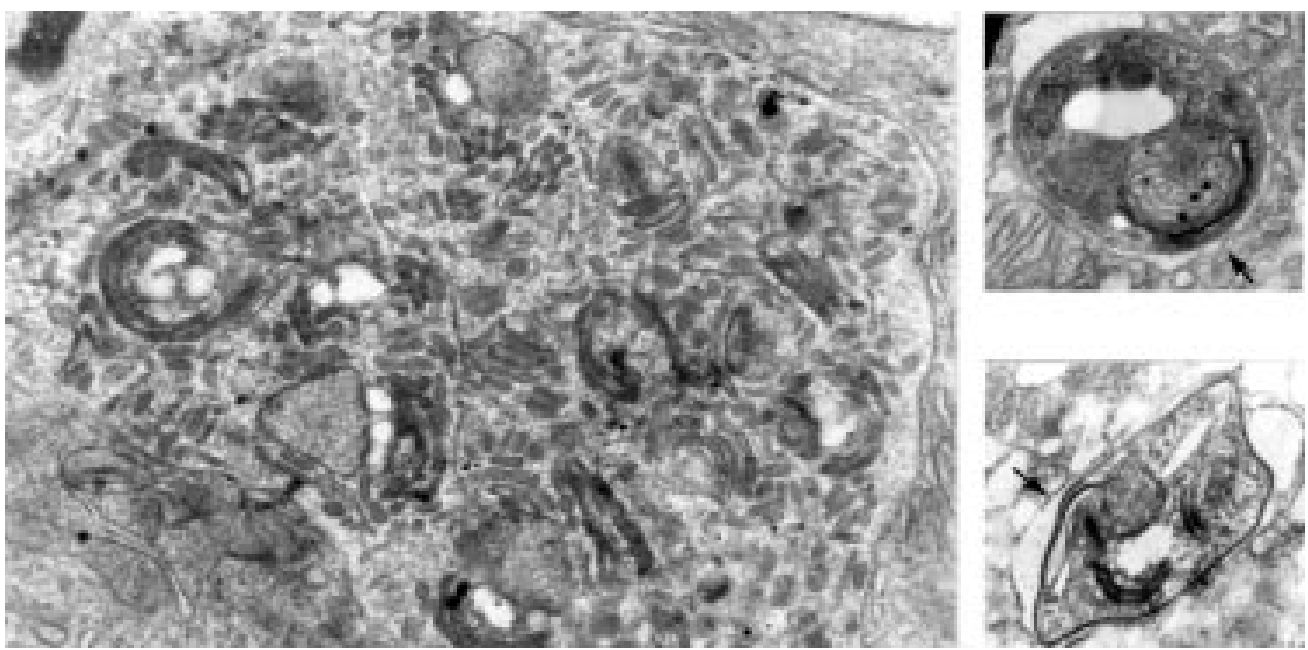

Figure 2. Normal sporogonial plasmodium (biopsy specimen before treatment). Dense nuclei with uniformly arranged chromatin are surrounded by electron-dense discs fused into arcs forming polar tube coils. (Magnification $\times 9000$.) Top right: Normal mature spore (biopsy specimen before treatment). There is a dense nucleus, a polaroplast (arrow), an electron-lucent vacuole, and a double row of coils of the polar tube. (Magnification $\times 5960$.) Bottom right: Normal sporoblast (biopsy specimen before treatment). A dense nucleus, an anchoring disc with associated polaroplast membranes (arrow), and some polar tube coils are seen. (Magnification $\times 11$ 170.)

Case 1 regained his preinfection body weight and was asymptomatic during four months of follow up, although very rare to frequent microsporidian spores, most of which were dysmorphous, were intermittently excreted in the stools. The patient later moved to another city and was lost to the follow up.

Case 2 was symptom-free over 15 months of follow up, and regained his preinfection body weight. He showed clearance of parasite in stool, with occasional shedding of very rare to frequent spores, most of which were dysmorphous.

Case 3 had six months of follow up showing parasite clearance in stool or intermittent shedding of very rare spores, most of which were dysmorphous. The patient regained his preinfection body weight and was asymptomatic until he died of Rhodococcus equi pneumonia and Staphylococcus aureus septicaemia.

Case 4 had no diarrhoea during seven months of follow up, although there was intermittent shedding in the stools of very rare to very frequent spores, almost all being dysmorphous. The patient showed body weight

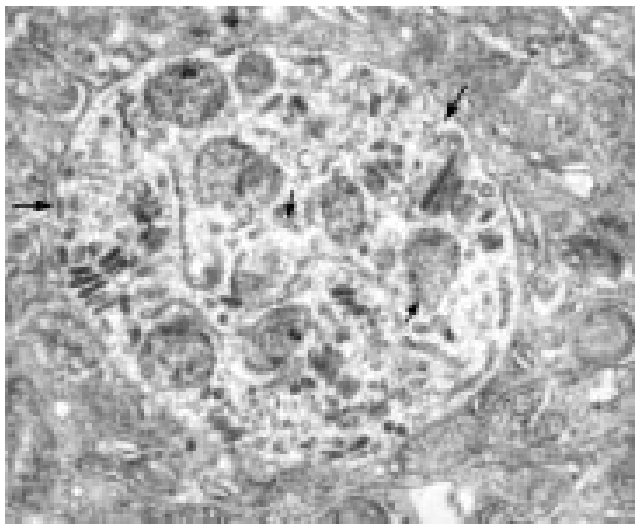

Figure 3 Sporogonial plasmodium (biopsy specimen after treatment). Nuclei are deformed with disarrangement of chromatin and disruption of nuclear membranes (arrows). Electron-dense discs do not fuse into arcs and appear disorganised; some are disrupted and pale (arrows). $($ Magnification $\times 6460$.) increase, and had neither clinical nor parasitological relapse until his death from severe intestinal bleeding.

PARASITE CHANGES IN THE STOOLS

Parasite changes were detected by Weber's stain in all patients' stools after treatment. In these cases a marked red stained clot was distinguishable within the spores (fig 1), and replaced the pinkish red belt-like stripe originally described by Weber et al. ${ }^{17}$ The mean number of altered spores was 10 in each microscopic field (range 1-50).

Dysmorphous spores were intermittently shed in stool, and coexisted with normal spores with a mean ratio of $5: 1$. These spore changes seen by light microscopy were persistently detected during follow up in all the patients, and were identical to those described after treatment with furazolidone alone. ${ }^{13}$

PARASITE DAMAGE IN BIOPSY SPECIMENS

Unaltered phases of the E bieneusi life cycle were found in all fields studied from biopsies

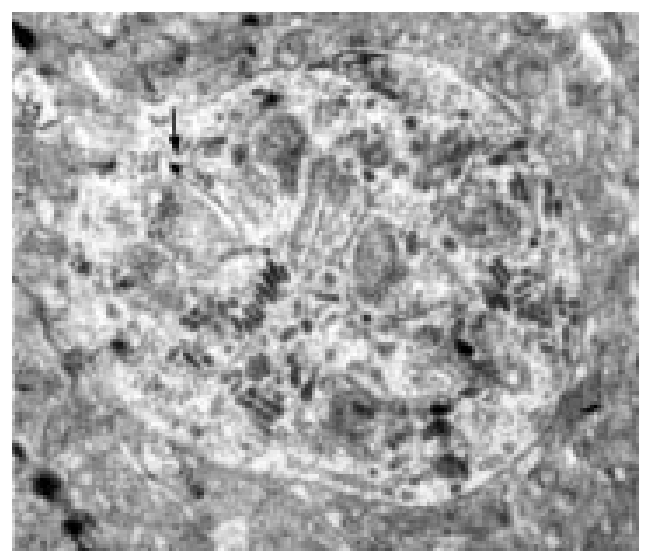

Figure 4 Sporogonial plasmodium (biopsy specimen after treatment). Vacuolated nuclei and separation of the nuclear membrane from the nucleoplasm are seen (arrow).

Electron-dense discs fail to develop into arcs and appear disorganised. (Magnification $\times 6460$.) 


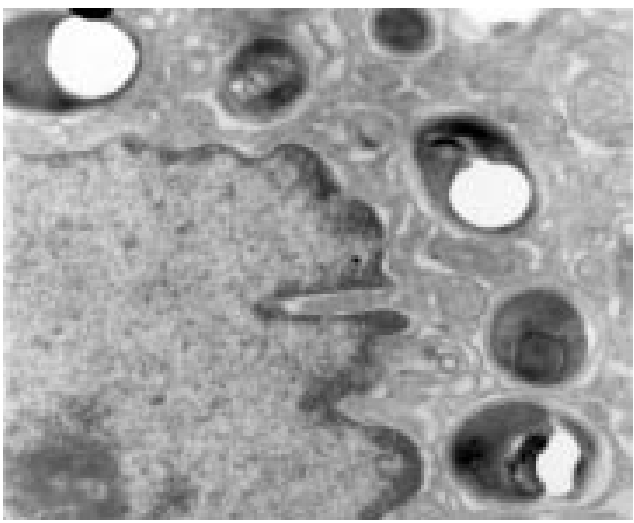

Figure 5 Mature spores (biopsy specimen after treatment). Osmiophilic spores are present, some containing a very enlarged vacuole. (Magnification $\times 9800$ ).

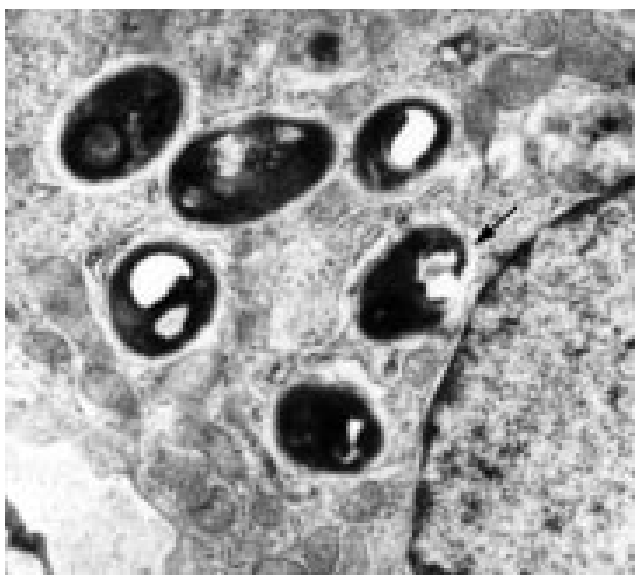

Figure 6 Mature spores (biopsy specimen after treatment). Very electron-dense spores are seen. One spore is broken (arrow). (Magnification $\times 6380$.).

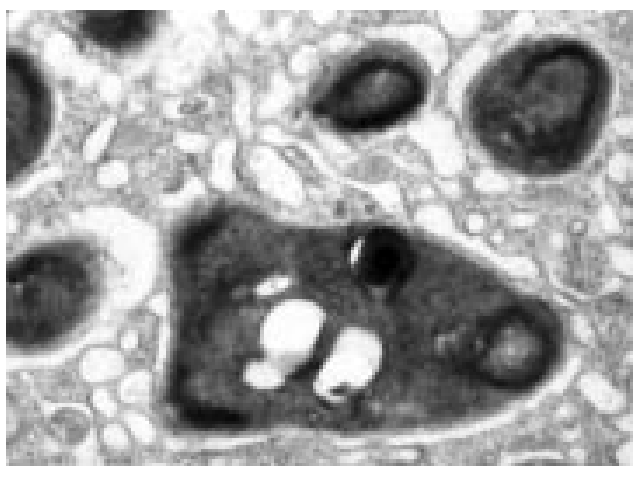

Figure 7 Giant spore (biopsy specimen after treatment). Two electron-lucent vacuoles, multiple polar tube coils, and a polaroplast are shown. (Magnification $\times 6570$.)

taken before treatment (fig 2), ${ }^{21}$ and the parasites were present in almost all of the semithin and ultrathin sections obtained.

After treatment, ultrastructural changes at all stages of $E$ bieneusi were observed in the biopsy specimens, and were most pronounced in sporogonial plasmodia and spores. Most sporogonial plasmodia had disarranged nuclear chromatin, and many showed disruption of nuclear membranes, irregularity of nuclear profiles and diastasis between the nuclear membrane and the nucleoplasm (figs 3 and 4). Several electron-dense discs also failed to develop into polar tubes and appeared disor- ganised, deformed, disrupted, and pale (figs 3 and 4). Most spores were very electron-dense, and showed enlarged electron-lucent vacuoles (fig 5).

These changes were observed both in biopsy specimens obtained just after completion of treatment and two months later, and were similar to changes reported after furazolidone alone. ${ }^{13}$

There was a substantial decrease in microsporidia, documented by both light microscopy and TEM, in all biopsies taken either just after completion of treatment or two months later, and the parasites were found in fewer than half of all semithin and ultrathin sections obtained. A broken spore and a giant spore containing two electron-lucent vacuoles and multiple coils of the polar tube were also observed in biopsies taken after treatment (figs 6 and 7).

These alterations were not found after treatment with furazolidone alone. ${ }^{13}$ No abnormalities in host cell structures were seen in biopsy specimens obtained before and after treatment.

\section{Discussion}

Combination treatment with furazolidone and albendazole was associated with a complete response in four severely immunocompromised AIDS patients with symptomatic E bieneusi infection.

All patients had long lasting symptomatic relief with a substantial decrease-or transient absence-of protozoan shedding in the stools, and regained their preinfection body weight. The regimen was well tolerated by all the patients, and there were no alterations in the laboratory test results attributable to the drugs used. During long term follow up no patient had a relapse, either clinical or parasitological, despite there being no documented improvement in CD4+T cell count and no maintenance treatment. Such a prolonged effect was not seen after treatment with furazolidone alone, ${ }^{13}$ nor has it been reported to date after any other treatment against $E$ bieneusi.

Combination treatment with furazolidone and albendazole resulted in persistent alterations in the parasite. Ultrastructural changes at all stages of $E$ bieneusi were documented not only in biopsy specimens taken at endoscopy just after completion of treatment, but also in biopsies obtained two months later. These changes were most pronounced in sporogonial plasmodia and spores, and were similar to changes observed after furazolidone alone, ${ }^{13}$ including osmiophilic spores with enlarged vacuoles as revealed in a retrospective TEM study.

A substantial decrease in microsporidia was a persistent feature of all biopsies obtained, either immediately after discontinuation of treatment or two months later. We also found both a broken spore and a giant spore, suggesting incomplete division of the sporonts. These findings are of interest as they were not observed after furazolidone alone, ${ }^{13}$ nor have they been reported with any other treatment, though giant spores have previously been found with albendazole treatment. ${ }^{11}{ }^{12}$ Furthermore, dysmorphous spores were persistently 
documented in all patients' stool by Weber's method after combination therapy, and were correlated to the post-treatment parasite alterations seen by TEM in biopsy specimens. These changes in faecal spores were identical to those previously described after furazolidone alone, ${ }^{13}$ and were not seen in our four patients before treatment. We hypothesise that these changes could derive from furazolidone mediated damage to the spore coat, with subsequent increasing permeability, as suggested by osmiophilic spores seen by TEM in biopsy specimens.

We are unable to explain why dysmorphous spores persisted for long periods in all the patients' stools after combination treatment was discontinued. We can only hypothesise that this might have been caused by the persistence of genomic damage to the parasite induced by furazolidone and transmissible, as an inherited defect, to the progeny during reproduction. This hypothesis agrees with our TEM results of persistent alterations in $E$ bieneusi, at all stages of the life cycle, in all biopsies obtained two months after stopping treatment.

Both the decrease of parasite load and the parasite damage seen in post-treatment biopsies may account for the decrease in parasite load and the spore changes persistently seen in stool after treatment. These findings are in agreement with a recent report that closely linked the quantitation of $E$ bieneusi in tissue with quantitation in the stools, ${ }^{22}$ and may explain the long lasting symptomatic relief we observed during follow up in all the patients.

Our preliminary results suggest that combined furazolidone and albendazole have a direct action on $E$ bieneusi, probably by inhibiting parasite multiplication, and may be effective in achieving persistent clinical and parasitological remission even in patients with severe immunodeficiency. Whether our results can be interpreted as an enhancement of albendazole activity secondary to parasite damage caused by furazolidone is difficult to assess at this stage, although a complementary effect, as suggested by clinical and parasitological responses, seems probable.

This study confirms the results reported after furazolidone alone, ${ }^{13}$ and emphasises the importance of combining furazolidone with albendazole to achieve a substantial and persistent reduction in the parasites, avoiding the need for maintenance treatment. However, although this combination greatly reduced the numbers of microsporidia, it did not completely eradicate the infection as some parasites developed normally and gave rise to normal spores. Nevertheless, our study supports the efficacy and safety of a new drug regimen for the treatment of $E$ bieneusi microsporidiosis. The long lasting symptomatic relief observed after combined furazolidone and albendazole was correlated with the persistent decrease in parasite burden, both in the tissues and in the stools, and with the morphological changes of the protozoan life cycle.

The long term natural history of $E$ bieneusi infection is not well understood, and spontaneous remission-as occurs with cryptosporidiosis-is quite often seen in clinical
Table 1 Concomitant infections

\begin{tabular}{lll}
\hline Case & Disease & Treatment \\
\hline 1 & None & None \\
2 & Oral candidiasis & Fluconazole \\
3 & $\begin{array}{l}\text { Oesophageal candidiasis } \\
\text { Rhodococcus equi } \\
\text { pneumonia }\end{array}$ & $\begin{array}{l}\text { Vluconazole } \\
\text { clarithromycin }+ \text { rifampicin }+\end{array}$ \\
& $\begin{array}{l}\text { Staphylococcus aureus } \\
\text { septicaemia }\end{array}$ & Vancomycin \\
4 & Oesophageal candidiasis & Fluconazole \\
\hline
\end{tabular}

practice. Randomised controlled trials are required to validate the results of our uncontrolled pilot study and to dispel doubts about spontaneous remission or improvement resulting from the treatment of concomitant infections. These factors were unlikely to have been operative in our cases, however, since all the patients showed a pronounced response to the combination treatment, while no spontaneous remission, either clinical or parasitological, had been observed before. Moreover, to our knowledge, none of the drugs we used to treat concomitant infections (table 1) has any reported activity against $E$ bieneusi.

We are indebted to Dr Esther Diana for constructive criticism and helpful comments in reviewing manuscript.

1 Bryan RT. Microsporidia. In: Mandell GL, Bennett JE, Dolin R, eds. Principles and practice of infectious diseases. New York: Churchill Livingstone, 1995:2513-24.

2 Curry A, Canning EU. Human microsporidiosis. I Infect Curry A, Canning
1993;27:229-36.

2b Molina JM, Gognel J, Sarfati C, et al. Potential efficacy of fumagillin in intestinal microsporidiosis due to Enterocytozoon bieneusi in patients with HIV infection: results of a drug screening study. AIDS 1997;11:1603-10

3 Sharpstone D, Rowbottom A, Francis N, et al. Thalidomide: a novel therapy for microsporidiosis. Gastroenterology 1997; 112:1823-9.

4 Anwar Bruni DM, Hogan SE, Schwartz DA, et al. Atovaquone is effective treatment for the symptoms of gastrointestinal microsporidiosis in HIV-1 infected patients. AIDS 1996;10:619-23.

5 Albrecht A, Stellbrink HJ, Sobottka I. Failure of itraconazole to prevent Enterocytozoon bieneusi infection. Genitourin Med 1995;71:325-6.

6 Soule JB, Halverson AL, Becker RB, et al. A patient with acquired immunodeficiency syndrome and untreated Encephalitozoon (Septata) intestinalis microsporidiosis leading to small bowel perforation. Response to albendazole. Arch Pathol Lab Med 1997;121:880-7.

7 Dore GJ, Marriott DJ, Hing MC, et al. Disseminated microsporidiosis due to Septata intestinalis in nine patients infected with the human immunodeficiency virus: response to therapy with albendazole. Clin Infect Dis 1995;21:70-6.

8 Joste NE, Rich JD, Busam KJ, et al. Autopsy verification of Encephalitozoon intestinalis (microsporidiosis) eradication following albendazole therapy. Arch Pathol Lab Med 1996;120:199-203.

9 Didier ES. Effects of albendazole, fumagillin, and TNP-470 on microsporidial replication in vitro. Antimicrob Agents Chemother 1997;41:1541-6.

10 Dieterich DT, Lew EA, Kotler DP, et al. Treatment with albendazole for intestinal disease due to Enterocytozoon bieneusi in patients with AIDS. F Infect Dis 1994;169:17883.

11 Blanshard C, Ellis DS, Dowell SP, et al. Electron microscopic changes in Enterocytozoon bieneusi following microscopic changes in Enterocytozoon bieneusi following 902 .

12 Voglino MC, Donelli G, Rossi L, et al. Intestinal microsporidiosis in Italian individuals with AIDS. Ital $\mathcal{f}$ Gastroenterol 1996;28:381-6.

13 Dionisio D, Ibba Manneschi L, Di Lollo S, et al. Enterocytozoon bieneusi in AIDS: symptomatic relief and parasite changes after furazolidone. $\mathcal{F}$ Clin Pathol 1997;50:472-6.

14 White AH. Absorption, distribution, metabolism and excretion of furazolidone. Scand $\mathcal{F}$ Gastroenterol 1989;24(suppl 169):4-10.

15 Haque MA, Hollister WS, Wilcox A, et al. The antimicrosporidial activity of albendazole. F Invert Pathol 1993;62: $171-7$.

16 Li J, Katiyar SK, Hamelin A, et al. Tubulin genes from AIDS-associated microsporidia and implications for phylogeny and benzimidazole sensitivity. Mol Biochem Parasitol 1996);78:289-95.

17 Weber R, Bryan RT, Owen RL, et al. Improved light microscopical detection of microsporidia spores in stools and duodenal aspirates. $N$ Engl f Med 1992;326:161-6. 
18 Van Gool T, Snijders F, Reiss P, et al. Diagnosis of intestinal and disseminated microsporidial infections in patients with HIV by a new rapid fluorescence technique. $\mathcal{F}$ Clin Pathol 1993;46:694-9.

19 Ignatius R, Henschel S, Liesenfeld O, et al. Comparative evaluation of modified trichrome and Uvitex $2 \mathrm{~B}$ stains for detection of low numbers of microsporidial spores in stool specimens. F Clin Microbiol 1997;35:2266-9.

20 Altamirano A, Bondani A. Adverse reactions to furazolidone and other drugs. A comparative review. Scand $\mathcal{F}$ Gastroenterol 1989;24(suppl 169):70-80.

21 Cali A Owen RL Int zoon, a uniqu. mitracellular development of EnterocytoAIDS patients. F Protozool 1990;37:145-55.

22 Clarridge JE, Karkhanis S, Rabeneck L, et al. Quantitative light microscopic detection of Enterocytozoon bieneusi in stool specimens: a longitudinal study of human immunodeficiency virus-infected microsporidiosis patients. I Clin Microbiol 1996;34:520-3.

\section{Royal College of Pathologists Forthcoming Symposia}

Transfusion Medicine Update

17-18 November 1998

Cytopathology Update

17 December 1998

What to do When the Cause of Death is in the Brain

27 January 1999

Antimicrobial Resistance: In Humans and Other Animals

17 February 1999

Cytokines and Chemokines in Disease

18 March 1999

Can't Give a Cause of Death-Won't Give a Cause of Death 22 April 1999

These meetings will be held at the Royal College of Pathologists, 2 Carlton House Terrace, London SW1Y 5AF and are open to members and non-members of the College.

Further details and application forms from the Scientific Meetings Officer, tel +44 (0)171930 5862, ext 24/25; fax +44 (0)1713210523. 